\title{
STUDIES ON IMPERFECTIONS IN THE SLS STORAGE RING
}

\author{
M. Böge, A. Streun, M. Muñoz, Paul Scherrer Institute, Switzerland
}

\begin{abstract}
Studies on linear and nonlinear imperfections in the SLS storage ring operated at $2.4 \mathrm{GeV}$ are presented. The influence of spurious vertical dispersion and linear coupling on the vertical emittance and possible correction schemes are discussed. The deterioration of the Dynamic Aperture caused by higher order multipoles is investigated based on field calculations. Furthermore the influence of ground waves on the orbit stability is estimated.
\end{abstract}

\section{THE MACHINE MODEL}

For a successful operation of the SLS storage ring [1] it is crucial to keep the tolerances on linear and nonlinear imperfections as small as possible. Correction schemes have to compensate for the remaining errors. In order to simulate the imperfect SLS ring a realistic machine model has been developed utilizing the 6D code TRACY[2][3].

Horizontal and vertical alignment errors, magnet tilts, strength distortions and higher order multipoles are included. The fact that the elements are mounted on girders and the dipoles are chaining adjacent girders introduces a correlation which has to be taken into account in the simulation. The elements on the girders are assumed to have an rms aligment error of $50 \mu \mathrm{m}$, the girders themselves $300 \mu \mathrm{m}$ and the girder joints $100 \mu \mathrm{m}$.

The roll of the girders is controlled to $25 \mu \mathrm{rad}$ by a hydrostatic leveling system. Individual elements are allowed to have residual rolls of $100 \mu \mathrm{rad} \mathrm{rms}$. In order to get a proper statistics 200 different error sequences (seeds) have been chosen in the simulation. It should be noted that all assigned errors are gaussian distributed with a cut at two sigma.

\section{ORBIT CORRECTION}

Once the errors have been assigned a first turn steering algorithm ("threader") is used to find the initial closed orbit. After setting the sextupoles to $50 \%$ of their strength a closed orbit correction is performed which is based on the information of beta functions and phases for the ideal optics. This is followed by another correction loop at full sextupole strength until the monitors have zero readings.

For the orbit correction two schemes are considered. One is based on the Singular Value Decomposition (SVD) algorithm. The other involves interleaved three corrector bumps "sliding" around the machine.

The global SVD scheme has the advantage of being able to handle an unequal number of monitors and correctors in the case of faulty monitors and/or saturated correctors and is therefore very flexible. On the other hand the

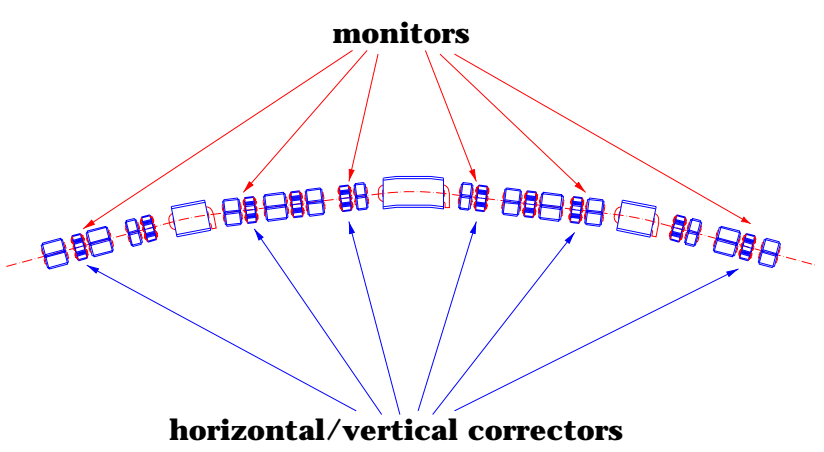

Figure 1: Corrector/Monitor layout of one sector (1/12th) of the SLS storage ring

SVD scheme requires a good knowledge of the linear machine optics in order to determine the inverse of the corrector/monitor correlation matrix $A^{-1}$.

For the envisaged monitor and corrector layout (72 monitors and 72 correctors at the same locations in both planes) (see Figure 1) the SVD and the sliding bump orbit correction scheme converge to the same correction state. This can be explained by the fact that $A^{-1}$ is a sparse tridiagonal matrix containing the kick ratios of interleaved three corrector bumps. It should be noted that the properties of $A^{-1}$ have implications on the implementation of the fast global orbit feedback [4].

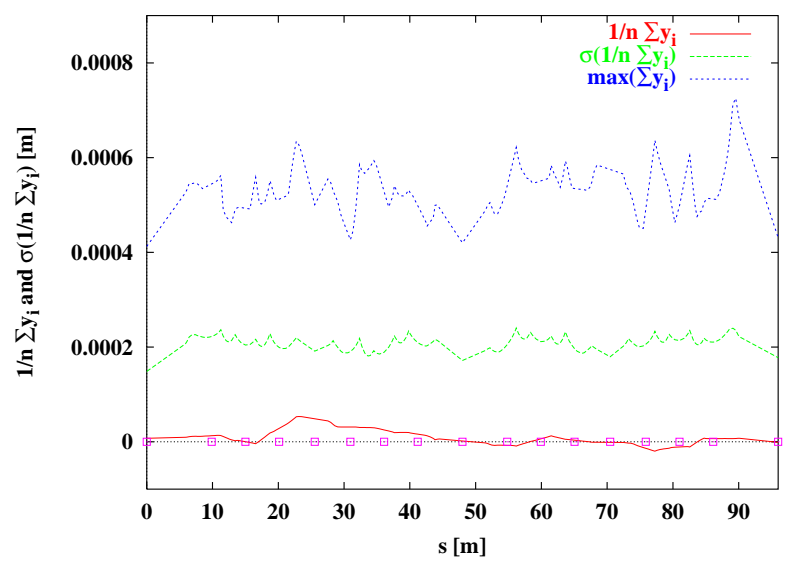

Figure 2: Mean, rms and maximum vertical orbit for 200 seeds for $1 / 3 r d$ of the lattice.

After the correction rms values of about $200 \mu \mathrm{m}$ (zero monitor readings) are observed in both planes. As an example Figure 2 visualizes the mean, rms and maximum orbit in the vertical plane. The maximum corrector kicks needed are $50 \%$ below the design maximum of $\approx 1 \mathrm{mrad}$. 
In the vertical direction about $20 \%$ more corrector strength is needed than in the horizontal plane although the rms horizontal kick is about $30 \%$ larger. This can be explained by a $50 \%$ less efficient correction in the vertical plane.

\section{COUPLING CORRECTION}
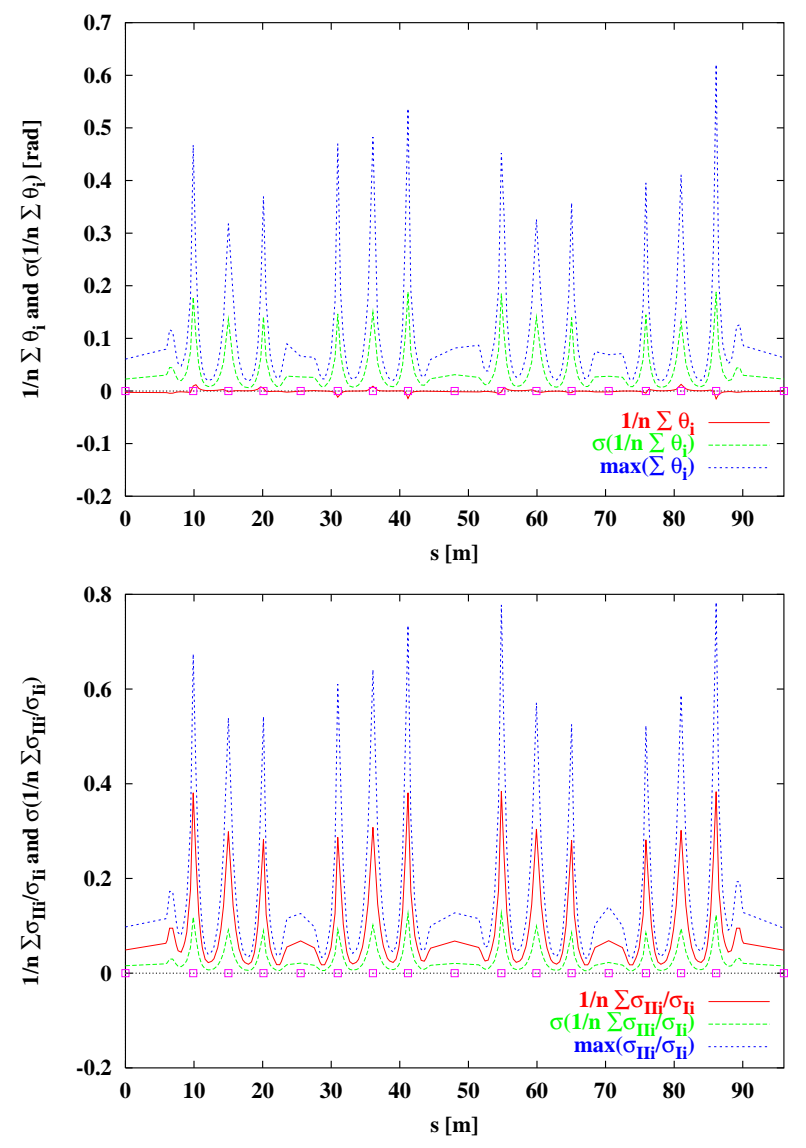

Figure 3: Top: Mean, rms and maximum beam ellipse twist $\theta$ for 200 seeds for $1 / 3$ rd of the lattice, bottom: corresponding sigma ratio $\sigma_{I I} / \sigma_{I}$.

Betatron and emittance coupling have been estimated from the calculation of generalized emittances and sigma matrices [5]. The beam ellipse twist in the straight sections of the SLS storage ring has been found to be $\approx 40 \mathrm{mrad}$ (see top graph of Figure 3).

The corresponding value for the emittance coupling in mode D1 which allows $5-8 \mathrm{~cm}$ horizontal dispersion in the straight sections is calculated to be $0.2 \%$ and $1 \%$ in the zero dispersion mode D0. This relatively large coupling factor for the latter mode can be explained by the fact that the vertical working point had been initially chosen very close to the integer $\left(\nu_{y}=7.08\right)$ in order to optimize the Dynamic Aperture. At the same time this results in a significant increase of the spurious vertical dispersion. A change of the vertical tune to $\nu_{y}=8.28$ (D2A lattice) leads to a reduction of the emittance coupling to $0.25 \%$. The bottom graph of Figure 3 shows the sigma ratios $\sigma_{I I} / \sigma_{I}$ for
$1 / 3$ rd of the D2A lattice after the tune change. The contribution from quadrupoles is nicely compensated by the dispersion generated by the nearly adjacent orbit correctors in the sextupoles. Thus the remaining vertical dispersion of $0.3 \mathrm{~cm}$ is mainly induced by sextupoles. Another source of emittance coupling is the feeddown of horizontal dispersion through skew quadrupole components induced by nonzero vertical orbits in sextupoles and quadrupole roll errors. This contribution can be minimized utilizing dedicated skew quadrupoles. Foreseen are three families with two magnets per family paired around the three long straight sections. The idea is to use the foreseen additional corrector windings on the sextupoles to generate the necessary field. The effectivness of the correction scheme has been tested for the 200 seeds. The resulting histograms for the emittance ratio $\kappa$ are depicted in Figure 4. It can be seen that the mean $\kappa$ value is reduced from $0.25 \%$ (see curve labeled sextupoles +tilt error+no correction) to $0.1 \%$ (see curve labeled sextupoles + tilt error+correction). Furthermore the $\kappa$ spread has been reduced from $0.16 \%$ to $0.06 \%$. It can be also deduced from the graph that magnet tilt errors have only a marginal influence on $\kappa$ (see curves labeled sextupoles + tilt error $+($ no) correction). Switching of the sextupoles results in a $\kappa$ of $0.02 \%$ (see curve labeled no sextupoles +tilt error+correction) which illustrates that the residual coupling is dominated by sextupoles. Quadrupoles plus correctors alone account for a $\kappa$ of $0.01 \%$ (see curve labeled no sextupoles+no tilt error).

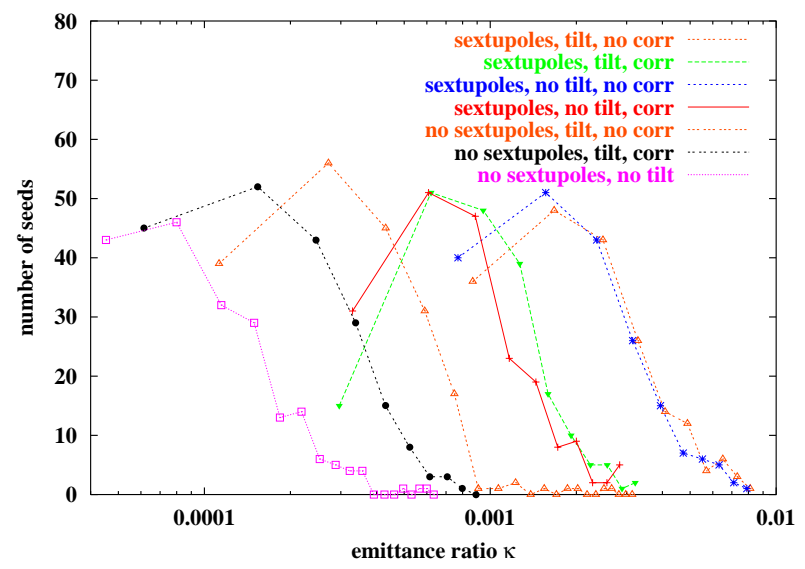

Figure 4: Histograms of the emittance ratio $\kappa$ for 200 seeds with and without coupling correction.

\section{MULTIPOLE ERRORS}

Results from 2D field calculations [11] for quadrupoles and dipoles have been investigated concerning the impact on Dynamic Aperture [10]. Alignment errors and orbit correction have been included. It has been found that the multipoles cut off all Dynamic Aperture outside the physical aperture which is simply due to the fact, that the multipole decomposition is only valid within the pole inscribed radius. Tracking including physical limits virtually does 
not show any Dynamic Aperture deterioration. Subsequent tests using data from 3D field calculations for quadrupoles and sextupoles have confirmed this result.

The vertical correctors with dipole coefficient $b_{1}$ which are integrated into the sextupoles create a large decapole component $b_{5}$. The ratio of the multipole coefficients $b_{5} / b_{1}$ is calculated to be $5.2510^{5} \mathrm{~m}^{-4}$. Assuming a linear scaling of $b_{5}$ with $b_{1}$ the Dynamic Aperture has been calculated for 200 distorted and orbit corrected machines operated in the D0 lattice mode. It has been shown that the Dynamic Aperture is only reduced for large momentum deviations $\mathrm{dp} / \mathrm{p}>2 \%$.

\section{GROUND WAVES}

The magnets of the SLS storage ring are mounted onto 48 girders with the 36 bending magnets of the 12 TBAs sitting on the ends of two adjacent girders [7]. The amplitude of stored beam orbit oscillation excited by groundwaves is calculated by convolution of the seismic spectrum on site, damping by the concrete slab of the hall, girder mechanical amplification factors, closed orbit distortion amplification factors and attenuation factors of the fast feedback system [4].

For a rough worst case estimate the following assumptions are made:

- The largest ground noise observed was recorded while a heavy truck was passing by on the nearby road. Peaks of $300 \mathrm{~nm}$ at $30 \mathrm{~Hz}$ and $30 \mathrm{~nm}$ at $60 \mathrm{~Hz}$ [8] were found. Continuos ground noise however is at least one order of magnitude lower.

- Within the worst case estimate the damping effect of the hall's $40 \mathrm{~cm}$ concrete slab are neglected.

- The mechanical amplification factors of the girder were measured to be $\leq 10$ on resonance horizontally and vertically over a frequency range from $0 . .50 \mathrm{~Hz}$ [9].

- Amplification factors for the closed orbit with and without girders are shown in Fig. 5. With girders one sees horizontal [vertical] amplification factors of 8 [5] at $30 \mathrm{~Hz}$ and 25 [5] at $60 \mathrm{~Hz}$.

- The attenuation provided by the fast feedback system is about $-55 \mathrm{~dB}$ at $30 \mathrm{~Hz}$ and $-35 \mathrm{~dB}$ at $60 \mathrm{~Hz}$ (assuming that there is only one dominant peak) [4].

Multiplying these numbers one arrives at rms average orbit distortions of 25 [15] $\mu \mathrm{m}$ horizontally [vertically] at $30 \mathrm{~Hz}$ and $7.5[1.5] \mu \mathrm{m}$ at $60 \mathrm{~Hz}$ without feedback. Including the feedback the orbit distortions are well below $1 \mu \mathrm{m}$ over the whole frequency range.

With this as the result of a worst case estimate we are looking forward to achieve a rather quiet beam at SLS.
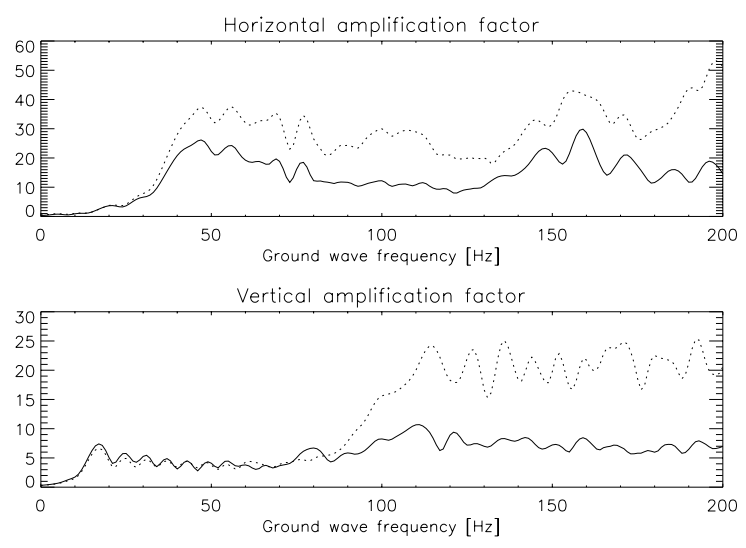

Figure 5: Amplification factors defined as ratio of closed orbit (averaged over ground wave incident angle and phase) to ground wave amplitude for single elements (dotted) and elements mounted on girders (solid). For high frequency the factors approach the values from random magnet misalignments which are for single elements, girder to girder and girder absolute alignment given by 58, 22, 6 horizontally and 25, 4, 3 vertically. An increase of the factors is observed where $\lambda$ equals the betatron wavelength, which occurs at $36 \mathrm{~Hz}$ for the horizontal and at $14 \mathrm{~Hz}$ for the vertical. Speed of sound was assumed to be $500 \mathrm{~m} / \mathrm{s}$ (soft ground).

\section{REFERENCES}

[1] M. Böge et al., "The Swiss Light Source Accelerator Complex: An Overwiew", Proc. of EPAC-98, Stockholm, (1998).

[2] J. Bengtsson, “TRACY-2 User's Manual”, Internal SLS document, (1997).

[3] M. Böge, "Update on TRACY-2 Documentation", SLSTME-TA-1999-0002, (1999).

[4] M. Böge et al., "Fast Closed Orbit Control in the SLS Storage Ring", Contribution to this Conference.

[5] A. W. Chao, J. Appl. Phys. 50, (1979).

[6] M. Böge et al., "Beam lifetime studies for the SLS storage ring", Contribution to this Conference.

[7] M. Böge et al., "SLS Lattice Finalization and Magnet Girder Design”, Proc. of EPAC-98, Stockholm, (1998).

[8] C. Markovits, "Erschütterungsmessungen des Schweiz. Erdbebendienstes am Ort der geplanten Synchrotronlichtquelle SLS auf dem Gelände des PSI West”, SLS-TME-TA-19940006, (1994).

[9] S. Zelenika, Private Communication.

[10] A. Streun, "SLS Dynamic Acceptance Degradation due to Magnet Multipole Errors", SLS-TME-TA-1998-0002, (1998).

[11] BINP Novosibirsk, Internal Design Report. 